Pacific Journal of Mathematics

PERIODIC POINTS ON TOR 


\section{PERIODIC POINTS ON TORI}

\section{BENJAMIN HALPERN}

\section{We prove the following theorem.}

Theorem 1. Given a continuous map $f: T^{n} \rightarrow T^{n}$ of the $n$-dimensional torus into itself. Each map homotopic to $f$ has an infinite number of periodic points if and only if the Lefschetz numbers of the iterates $L\left(f^{m}\right), m=1,2, \cdots$, are unbounded.

The "if" direction of Theorem 1 follows from a theorem of Brooks, Brown, Pak, and Taylor [1]. Let $N(f)$ denote the Nielsen number of the map $f$. Recall that each map homotopic to $f$ must have at least $N(f)$ distinct fixed points.

THeOREM 2. (Brook, Brown, Pak, and Taylor [1]). If $f: T^{n} \rightarrow T^{n}$ is a continuous map, then $N(f)=|L(f)|$.

The converse direction of Theorem 1 is deduced from the more precise result, Theorem 3.

Definition 1. Given a map $f: T^{n} \rightarrow T^{n}$. Let $\lambda_{1}, \cdots, \lambda_{n}$ be the characteristic values of $H_{1}(f): H_{1}\left(T^{n}\right) \rightarrow H_{1}\left(T^{n}\right)$. If $\lambda_{i}$ is not a root of unity, then set $a_{i m}=\left|1-\lambda_{i}^{m}\right|$. If $\lambda_{i}$ is a root of unity, then let $N$ be such that $\lambda_{i}^{N}=1$ and $\lambda_{i}^{m} \neq 1$ for $1 \leqq m<N$ (i.e., $\lambda_{i}$ is a primitive $N$ th root of unity), and set

$$
a_{i m}= \begin{cases}\left|1-\lambda_{i}^{m}\right| & \text { if } m \neq 0 \bmod N \\ \sum_{q \mid N}\left|1-\lambda_{i}^{q}\right| & \text { if } m \equiv 0 \bmod N .\end{cases}
$$

Set $a_{m}(f)=\prod_{\imath=1}^{n} a_{i m}$.

THEOREM 3. For each map $f: T^{n} \rightarrow T^{n}$ there exists a smooth map $g$ homotopic to $f$ such that for $m \geqq 1$,

$$
\#\left\{x \in T^{n} \mid g^{m}(x)=x\right\} \leqq a_{m}(f) \text {. }
$$

Since $L\left(f^{m}\right)=\prod_{i=1}^{n}\left(1-\lambda_{i}^{m}\right)$, we see that $\#\left\{x \in T^{n} \mid g^{m}(x)=x\right\}=N(f)$ for all $m$ such that $\lambda_{i}^{m} \neq 1$ for all $i$. From Theorems 2 and 3 , one may also deduce similarities between the asymptotic behaviors of $P_{m}=\#\left\{x \in T^{n} \mid g^{m}(x)=x\right\}$ and $Q_{m}=\max \left\{N\left(f^{r}\right) \mid 1 \leqq r \leqq m\right\}$.

In the process of proving Theorem 3 we establish a general result, Theorem 4, which concerns periodic points for maps homotopic to 
periodic maps.

THEOREM 4. Given a smooth compact connected manifold $M$ of dimension $m \geqq 2$, and a smooth map $f: M \rightarrow M$ such that $f^{N}=1_{M}$ for some $N \geqq 2$, and $f\left(x_{0}\right)=x_{0}$ for some $x_{0} \in M$. Also suppose $P=$ $\left\{x \in M \mid f^{r}(x)=x\right.$ for some $\left.r, 1 \leqq r<N\right\}$ is finite. Then there exists a smooth map $g: M \rightarrow M$ which is homotopic to $f$ and such that $P=$ the set of all periodic points of $g$, and $g|P=f| P$.

When Theorem 4 is specialized to tori, it gives a map $g$ homotopic to the given periodic map $f: T^{n} \rightarrow T^{n}$, whose numbers of periodic points of various periods are exactly the lower bounds implied by Theorem 2. Theorem 3 for an arbitrary map $f: T^{n} \rightarrow T^{n}$ is proved by homotoping $f$ to a map $g$ which with a "change of coordinates" takes the form $g: T^{n}=T^{k} \times T^{n-k} \rightarrow T^{k} \times T^{n-k}=T^{n}, g(x, y)=(a(x), r(x, y))$ where $a: T^{k} \rightarrow T^{k}$ is periodic. We homotopy $a$ to an $\bar{a}$ according to Theorem 4 and then, using an induction hypothesis we homotopy $r$ on the sets $\{x\} \times T^{n-k}$ for $x$ a periodic point of $\bar{a}$. This gives a map whose periodic points are the same as a map of the form $\bar{a} \times \bar{b}: T^{k} \times$ $T^{n-k} \rightarrow T^{k} \times T^{n-k}$ where $\bar{a}: T^{k} \rightarrow T^{k}$ and $\bar{b}: T^{n-k} \rightarrow T^{n-k}$. This is sufficient to prove Theorem 3 by induction, but it gives a map with possibly more periodic points than the lower bound set in Theorem 2 . In special cases the lower bound in Theorem 2 can be achieved by refinements in the technique outlined above. So we make the following conjecture.

Conjecture. Given a map $f: T^{n} \rightarrow T^{n}$. Then there exists a smooth map $g$ homotopic to $f$ such that $\#\left\{x \in T^{n} \mid x\right.$ is a periodic point of $g$ of least period $m\}=r_{m}$ where $r_{1}=|L(f)|$ and for $q \geqq 2$

$$
r_{q}= \begin{cases}0 & \text { if } L\left(f^{q}\right)=0 \\ \left|L\left(f^{q}\right)\right|-\sum_{\substack{m<q \\ m ! q}} r_{m} & \text { if } L\left(f^{q}\right) \neq 0 .\end{cases}
$$

This work was motivated by a question of Shub and Sullivan which appears on page 140 of Hirsch [3]. Shub and Sullivan ask whether every map homotopic to $g: T^{2} \rightarrow T^{2}$ must have an infinite number of periodic points where $g$ is the map covered by the linear map $\bar{g}: R^{2} \rightarrow R^{2}$ whose matrix is $\left(\begin{array}{ll}2 & 1 \\ 1 & 1\end{array}\right)$. Since the Lefschetz numbers $L\left(g^{n}\right)$ are easily seen to be unbounded, a positive answer follows from the theorem of Brooks, Brown, Pak, and Taylor, Theorem 2. An elementary, transparent proof of a special case of Theorem 2 is presented in Proposition 1.

2. Preliminaries. Denote the integers by $\boldsymbol{Z}$, the rationals by 
$\boldsymbol{Q}$, and the reals by $\boldsymbol{R}$. For each $a \in \boldsymbol{R}^{n}$ let $T_{a}: \boldsymbol{R}^{n} \rightarrow \boldsymbol{R}^{n}$ denote the translation by $a, T_{a} b=b+a$ for $b \in R^{n}$. Set $\mathscr{T}=\left\{T_{a} \mid a \in Z^{n}\right\}$. Let $\pi: \boldsymbol{R}^{n} \rightarrow T^{n}$ denote the usual covering map which identifies $T^{n}$ with $\boldsymbol{R}^{n} / \mathcal{S}$. Recall that an $n \times n$ matrix $A$ is unimodular provided it has integer entries and $\operatorname{det} A= \pm 1$, or equivalently, it has integer entries and an inverse with integer entries. Clearly, the rows of a matrix $A$ with integer entries form a basis for the module $Z^{n}$ over $Z$ if and only if $A$ is unimodular.

We will use the form of Nielsen fixed point theorem which states that if $f: X \rightarrow X$ is a continuous map of a compact manifold $X$ into itself, then each map $g$ homotopic to $f$ must have at least $N(f)$ fixed points, where $N(f)$ is the Nielsen number of $f$. Furthermore, $N(g)=$ $N(f)$, (Brown [2]). The Nielsen number $N(f)$ is defined as follows. First an equivalence relation $\sim$ is defined on the set $F$ of fixed points of $f$. Two fixed points $x, y \in F$ are equivalent, $x \sim y$, provided there is a path $\gamma$ in $X$ from $x$ to $y$ such that $f \circ \gamma$ is end points fixed homotopic to $\gamma$. The set of equivalence classes $F / \sim$ is known to be finite and each equivalence class is compact.

Using a fixed point index $I$, such as defined in [2] we may assign an index $i(A)$ to each $A \in F / \sim$ by setting $i(A)=I(U)$ for any open set $U$ such that $F \cap U=A$. The Nielsen number $N(f)$ is the number of $A \in F / \sim$ such that $i(A) \neq 0$. If $A$ is a singleton $\{x\}$, then $i\{x\}$ is the usual index of an isolated fixed point of $f$ and consequently if $f$ is differentiable and $1-d f_{x}$ is nonsingular, then $i\{x\}= \pm 1$ as $\operatorname{det}\left(1-d f_{x}\right)$ is positive or negative.

Let $e^{1}=(1,0, \cdots, 0), e^{2}=(0,1, \cdots, 0)$, etc., denote the standard basis for $\boldsymbol{R}^{n}$. Set $\beta_{i}(t)=\pi\left(t e^{i}\right)$ and $\alpha_{i}=\left[\beta_{i}\right] \in \pi_{1}\left(T^{n}, *\right)$, where $*=$ $\pi(0)$. Then $\alpha_{1}, \cdots, \alpha_{n}$ form a basis for $\pi_{1}\left(T^{n}, *\right)$. Since the Hurewitz homomorphism $\rho: \pi_{1}\left(T^{n}, *\right) \rightarrow H_{1}\left(T^{n}\right)$ is an isomorphism, we can identify $\pi\left(T^{n}, *\right)$ with $H_{1}\left(T^{n}\right)$ via $\rho$ and consider $\alpha_{1}, \cdots, \alpha_{n}$ as a basis for $H_{1}\left(T^{n}\right)$, which we shall call the standard basis of $H_{1}\left(T^{n}\right)$. If $L: R^{n} \rightarrow$ $\boldsymbol{R}^{n}$ is a linear map, we denote its matrix with respect to the standard basis by $\bar{L}$ and define it by $L\left(e^{i}\right)=\sum_{j} \bar{L}_{j i} e^{j}$. Throughout this paper we will consider $\boldsymbol{R}^{n}$ to be a space of column vectors. Then $\bar{L}$ satisfies $L(v)=\bar{L} v$ for all $v$ in $R^{n}$.

Consider the case where $\bar{L}_{j k} \in \boldsymbol{Z}$ for all $j, i$. Then for $a \in \boldsymbol{Z}^{n}$, $L a=b \in Z^{n}$. Since $L \circ T_{a}=T_{b} \circ L$, we see that $L$ induces a map $L^{\prime}: T^{n} \rightarrow T^{n}$. We say that $L$ covers $L^{\prime}$. It is a straightforward verification that the matrix of $H_{1}\left(L^{\prime}\right): H_{1}\left(T^{n}\right) \rightarrow H_{1}\left(T^{n}\right)$ with respect to the standard basis is equal to $\bar{L}$. Since $T^{n}$ is covered by $\boldsymbol{R}^{n}, T^{n}$ is an Eilenberg-MacLane space, $T^{n}=K\left(Z^{n}, 1\right)$. Hence the homotopy class of a map $f: T^{n} \rightarrow T^{n}$ is determined by the homomorphism $H_{1}(f): H_{1}\left(T^{n}\right) \rightarrow H_{1}\left(T^{n}\right)$. We sum up these observations in the following lemma. 
LemMa 1. Each map $f: T^{n} \rightarrow T^{n}$ is homotopic to a map $g: T^{n} \rightarrow$ $T^{n}$ which is covered by a linear map $\bar{g}: \boldsymbol{R}^{n} \rightarrow \boldsymbol{R}^{n}$ whose matrix is the same as the matrix of $H_{1}(f): H_{1}\left(T^{n}\right) \rightarrow H_{1}\left(T^{n}\right)$.

LEMMA 2. If $f: T^{n} \rightarrow T^{n}$ is covered by a linear map $A: \boldsymbol{R}^{n} \rightarrow \boldsymbol{R}^{n}$, $f \circ \pi=\pi \circ A$, and 1 is not a characteristic root of $A$, then the fixed points are isolated, they all have the same index, and the number of them is $|L(f)|$.

Proof. Let $x$ be a fixed point of $f$. Using an appropriate restriction of $\pi: R^{n} \rightarrow T^{n}$ for a coordinate system about $x$, we see that $d f_{x}$ expressed in these coordinates is $A$. Since $\operatorname{det}(1-A)=\prod_{i=1}^{n}\left(1-\lambda_{i}\right)$, where $\lambda_{1}, \cdots, \lambda_{n}$ are the characteristic roots of $A$, we see that $\operatorname{det}(1-A) \neq 0$. Hence $x$ is an isolated fixed point. Therefore, $i(x)=$ \pm 1 as $\operatorname{det}(1-A)$ is positive or negative, and so $i(x)$ is independent of $x$. The Lefschetz fixed point formula asserts that the sum of the $i(x)$ as $x$ ranges over the fixed points of $f$ is $L(f)$. Hence the number of fixed points is $|L(f)|$.

Proposition 1. Given a map $f: T^{n} \rightarrow T^{n}$ such that 1 is not a characteristic root of $H_{1}(f): T^{n} \rightarrow T^{n}$. Then $N(f)=|L(f)|$.

Proof. By Lemma 1 and the homotopy invariance of $N(f)$, we see that we may assume that $f$ is covered by a linear map $A: \boldsymbol{R}^{n} \rightarrow$ $\boldsymbol{R}^{n}$ and that 1 is not a characteristic root of $A$. From Lemma 2 we know that the set $F$ of fixed points of $f$ satisfies $\# F=|L(f)|$, and $i(x) \neq 0$ for each $x \in F$. To prove the present proposition it is sufficient to show that if $x, y \in F$, and $x \neq y$, then $x$ is not Nielsen equivalent to $y$. For then, each $\{x\}$ with $x \in F$ will be a distinct Nielsen equivalence class and their number, $\# F=|L(f)|$, will be equal to $N(f)$ by the definition of $N(f)$.

Assume $x, y \in F, x \neq y$, and $x \sim y$. Then there is a path $\gamma$ in $T^{n}$ from $x$ to $y$ such that $\gamma$ is end points fixed homotopic to $f \circ \gamma$. Let $\tilde{\gamma}: I \rightarrow \boldsymbol{R}^{n}$ be a lift of $\gamma, \pi \circ \widetilde{\gamma}=\gamma$, going from $\widetilde{\gamma}(0)=\widetilde{x}$ to $\widetilde{\gamma}(1)=\widetilde{y}$. Then $A \circ \tilde{\gamma}$ covers $f \circ \gamma$, since $\pi \circ A=f \circ \pi$. Set $a=\widetilde{x}-A(\tilde{\gamma}(0))=\tilde{x}-$ $A(\widetilde{x})$. Since $\pi(A(\widetilde{x}))=f(\pi(\widetilde{x}))=f(x)=x$ and $\pi(\widetilde{x})=x$ we deduce that $a \in Z^{n}$. Then $\pi \circ T_{a}=\pi$ and so $T_{a} \circ A \circ \tilde{\gamma}$ is also a lift of $f \circ \gamma$, $\pi \circ T_{a} \circ A \circ \tilde{\gamma}=\pi \circ A \circ \widetilde{\gamma}=f \circ \pi \circ \tilde{\gamma}=f \circ \gamma$. Also note that $T_{a} \circ A \circ \tilde{\gamma}(0)=$ $A(\tilde{\gamma}(0))+a=\tilde{x}=\tilde{\gamma}(0)$. Since $\gamma$ is end point fixed homotopic to $f \circ \gamma$, we have $T_{a} \circ A \circ \tilde{\gamma}(1)=\tilde{\gamma}(1)$. Therefore $\tilde{\gamma}(1)-\tilde{\gamma}(0)=T_{a} A \tilde{\gamma}(1)-T_{a} A \tilde{\gamma}(0)=$ $(A \tilde{\gamma}(1)+a)-(A \tilde{\gamma}(0)+a)=A(\tilde{\gamma}(1)-\tilde{\gamma}(0))$. From $x \neq y$ and $\pi \tilde{\gamma}(0)=x$ and $\pi(\widetilde{\gamma})(1)=y$, we conclude that $\tilde{\gamma}(0) \neq \tilde{\gamma}(1)$. Hence $\tilde{\gamma}(1)-\widetilde{\gamma}(0)$ is an eigenvector of $A$ with eigenvalue 1 , a contradiction. 


\section{Algebraic lemmas.}

LEMMA 3. Given $v=\left(v_{1}, \cdots, v_{n}\right) \in Z^{n}, n \geqq 1$, such that g.c.d. $\left(v_{1}\right.$, $\left.\cdots, v_{n}\right)=1$, where g.c.d. stands for greatest common divisor. Then there exist $v^{2}, v^{3}, \cdots, v^{n} \in Z^{n}$ such that $v, v^{2}, \cdots, v^{n}$ form a basis for $Z^{n}$.

Proof. We use induction on $n$. For $n=1$ we must show that $\{v\}$ is a basis for $Z^{1}$, i.e., that $v= \pm 1$. But this follows from the fact that g.c.d. $(v)=1$.

Now suppose $n>1$ and that the lemma holds for $n-1$. If $v_{1}=$ $v_{2}=\cdots=v_{n-1}=0$, then $v_{n}= \pm 1$ and the lemma obviously holds. So suppose that not all $v_{1}, v_{2}, \cdots, v_{n-1}$ are 0 . Let $d=$ g.c.d. $\left(v_{1}, \cdots, v_{n-1}\right)$. Then g.c.d. $\left(d, v_{n}\right)=1$ and so we may find $\alpha, \beta \in Z$ such that $\alpha v_{n}+$ $\beta d=1$. Apply the induction hypotheses to the vector $w=\left(v_{1} / d, \cdots\right.$, $\left.v_{n-1} / d\right) \in Z^{n-1}$ and obtain vectors $w^{2}, \cdots, w^{n-1} \in Z^{n-1}$ such that $w, w^{2}$, $\cdots, w^{n-1}$ form a basis for $Z^{n-1}$. Thus the matrix $A$ with rows $w, w^{2}, \cdots, w^{n-1}$ is unimodular, and so $\operatorname{det} A= \pm 1$. Let $B$ be the matrix with rows $d w, w^{2}, w^{3}, \cdots, w^{n-1}$. Then $\operatorname{det} B=d(\operatorname{det} A)$ and the first row of $B$ is $d w=\left(v_{1}, v_{2}, \cdots, v_{n-1}\right)$. Set $w^{i}=\left(w_{1}^{i}, \cdots, w_{n-1}^{i}\right)$ for $2 \leqq i \leqq n-1$. Form the matrix $C$ indicated below.

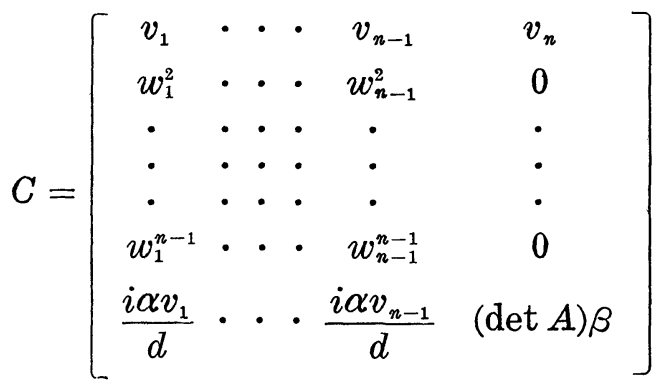

where $i=-\operatorname{det} A$. Then expanding $\operatorname{det} C$ on the last column we find $\operatorname{det} C=\alpha v_{n}+\beta d=1$. Thus the last $n-1$ rows satisfy the lemma.

LEMmA 4. If $v^{1}, v^{2}, \cdots, v^{r} \in Z^{n}, n \geqq 1$, then there is a unimodular matrix $A$ with rows $A^{1}, \cdots, A^{n}$ such that $\operatorname{sp}\left\{A^{1}, \cdots, A^{q}\right\}=\operatorname{sp}\left\{v^{1}, \cdots, v^{r}\right\}$, where $\mathrm{sp} V=$ the linear span in $\boldsymbol{R}^{n}$ for $V \subset \boldsymbol{R}^{n}$, and $q=\operatorname{dim} \operatorname{sp}\left\{v^{1}\right.$, $\left.\cdots, v^{r}\right\}$.

Proof. We may suppose $v^{1}, \cdots, v^{r}$ are linearly independent in $\boldsymbol{R}^{n}$. We will use induction on $r$. For $r=1$, Lemma 3 with $v=d^{-1} v^{1}$ where $v^{1}=\left(v_{1}^{1}, \cdots, v_{n}^{1}\right)$ and $d=$ g.c.d. $\left(v_{1}^{1}, \cdots, v_{n}^{1}\right)$ gives the desired conclusion.

Suppose now that $r \geqq 2$ and that Lemma 4 holds for $r-1$. Apply this supposition to $v^{1}, \cdots, v^{r-1}$ and obtain a unimodular matrix 
$B$ such that its rows $B^{1}, \cdots, B^{n}$ satisfy $\operatorname{sp}\left\{B^{1}, \cdots, B^{r-1}\right\}=\operatorname{sp}\left\{v^{1}, \cdots\right.$, $\left.v^{r-1}\right\}$. Note that $\operatorname{sp}\left\{B^{1}, \cdots, B^{r-1}, v^{r}\right\}=\operatorname{sp}\left\{v^{1}, \cdots, v^{r-1}, v^{r}\right\}$.

By considerring the linear transformation from $\boldsymbol{R}^{n}$ to $\boldsymbol{R}^{n}$ whose matrix is $B$ it is easily seen that it is sufficient to prove the lemma in the special case where $B^{i}=e^{i}$. Now let $v^{r}=\left(\alpha_{1}, \cdots, \alpha_{n}\right)$, and set

$$
w=\left(0, \cdots, 0, \alpha_{r}, \alpha_{r+1}, \cdots, \alpha_{n}\right)=v^{r}-\left(\alpha_{1}, \cdots, \alpha_{r-1}, 0, \cdots, 0\right) .
$$

Then $\operatorname{sp}\left\{B^{1}, \cdots, B^{r-1}, v^{r}\right\}=\operatorname{sp}\left\{B^{1}, \cdots, B^{r-1}, w\right\}$. Since $v^{r}$ is independent of $B^{1}, \cdots, B^{r-1}$, not all of $\alpha_{r}, \alpha_{r+1}, \cdots, \alpha_{n}$ can vanish. Set $d=$ g.c.d. $\left\{\alpha_{r}, \alpha_{r+1}, \cdots, \alpha_{n}\right\}$, and $u=d^{-1} w$. Then $u \in\{0\} \times Z^{n-r+1}$ and the greatest common divisor of its coordinates is 1 . Hence by Lemma 3 there is an $(n-r+1) \times(n-r+1)$ unimodular matrix $C$ such that the first row is $\alpha_{r} / d, \cdots, \alpha_{n} / d$. Set

$$
A=\left[\begin{array}{c|c}
I^{(r-1) \times(r-1)} & 0 \\
\hline 0 & C
\end{array}\right] .
$$

Then $A$ is unimodular, and its first $r$ rows are $B^{1}, \cdots, B^{r-1}, u$ which have the same span in $\boldsymbol{R}^{n}$ as does $v^{1}, \cdots, v^{r}$.

Suppose $f: T^{n} \rightarrow T^{n}, n \geqq 1$, is a map and $A$ is the matrix of $H_{1}(f): H_{1}\left(T^{n}\right) \rightarrow H_{1}\left(T^{n}\right)$. It is shown in [1] that $L(f)=\operatorname{det}\left(1-A^{t}\right)=$ $\operatorname{det}(1-A)$, where $A^{t}=$ the transpose of $A=$ the matrix of $H^{1}(f)$ : $H^{1}\left(T^{n}\right) \rightarrow H^{1}\left(T^{n}\right)$. Let $\lambda_{1}, \cdots, \lambda_{n}$ be the characteristic roots of $A$. Then $L(f)=\prod_{i=1}^{n}\left(1-\lambda_{i}\right)$. Since $\lambda_{1}^{m}, \cdots, \lambda_{n}^{m}$ are the characteristic roots of $A^{m}$ we have proved the following formula.

$$
L\left(f^{m}\right)=\prod_{i=1}^{n}\left(1-\lambda_{i}^{m}\right) .
$$

LEMma 5. Let $\lambda_{1}, \cdots, \lambda_{n}$ be complex numbers, none of them 1 , such that the set $\left\{\Pi_{i=1}^{n}\left|1-\lambda_{i}^{m}\right| \mid m=1,2, \cdots\right\}$ is bounded, then $\left|\lambda_{i}\right| \leqq 1$ for all $i=1, \cdots, n$.

Proof. Suppose not. Divide $\{1, \cdots, n\}$ into four sets $I, J, K$, and $L$ by setting $I=\left\{i|| \lambda_{i} \mid>1\right\}, J=\left\{i|| \lambda_{i} \mid<1\right\}, K=\left\{i \mid \lambda_{i}\right.$ is a root of unity\}, and $L=\left\{i|| \lambda_{i} \mid=1\right.$ and $\lambda_{i}^{q} \neq 1$ for all $\left.q \geqq 1\right\}$. For any $U \subset\{1, \cdots, n\}$, set $U_{m}=\Pi_{i \in U}\left|1-\lambda_{i}^{m}\right|$, with the convention that if $U=\varnothing$, then $U_{m}=1$ for all $m$. Formula $(*)$ gives $\left|L\left(f^{m}\right)\right|=I_{m} J_{m} K_{m} L_{m}$. Since $I \neq \varnothing$ we clearly have

$$
I_{m} \longrightarrow \infty \text {. }
$$


For each $i \in K$, let $q_{i} \geqq 1$ be such that $\lambda_{i}^{q_{i}}=1$ and $\lambda_{i}^{m} \neq 1$ for $1 \leqq m<q_{i}$. If $K \neq \varnothing$, set $q=\prod_{i \in K} q_{i}$. If $K=\varnothing$, set $q=1$. In either case, for $p \geqq 0$,

$$
K_{q p+1}=K_{1}>0 \text {. }
$$

Here we have used the hypothesis that $\lambda_{i} \neq 1$ for all $i$.

Set $N=\# L$. From the definition of $L$ we see that $1 \notin\left\{\lambda_{i}^{q r} \mid i \in L\right.$, $r=1, \cdots, N\}$, and hence we can find an $\varepsilon>0$ such that $\left|1-\lambda_{i}^{q r}\right|>2 \varepsilon$ for $i \in L$ and $1 \leqq r \leqq N$.

Claim. For each $i \in L$, and each positive integer $a$, at most one member of the sequence $\lambda_{i}^{q m+1}$, where $a(N+1)<m \leqq(a+1)(N+1)$, satisfies

$$
\left|1-\lambda_{i}^{q m+1}\right| \leqq \varepsilon
$$

Proof. Suppose not. Then there is an $i \in L, m$ and $r$ such that $1 \leqq r \leqq N$, and $\left|1-\lambda_{i}^{q m+1}\right| \leqq \varepsilon$, and $\left|1-\lambda_{i}^{q(m+r)+1}\right| \leqq \varepsilon$. It follows that

$$
\begin{aligned}
2 \varepsilon \geqq\left|\lambda_{i}^{g(m+r)+1}-\lambda_{i}^{q m+1}\right| & =\left|\lambda_{i}^{q m+1}\right|\left|\lambda_{i}^{q r}-1\right| \\
& =\left|\lambda_{i}^{q r}-1\right|>2 \varepsilon,
\end{aligned}
$$

a contradiction. This proves the claim.

Since the number of $m$ 's which satisfy $a(N+1)<m \leqq(a+1)(N+1)$ is $N+1$ and $N=\# L$, for each $a$ there is an $m$ such that $a(N+1)<$ $m \leqq(a+1)(N+1)$, and $\left|1-\lambda_{i}^{q m+1}\right|>\varepsilon$ for all $i \in L$. Hence $L_{m q+1} \geqq \varepsilon^{N}$ for an infinite number of $m$ 's. Note that this also holds when $N=0$. Combining this with (1), (2), and (3) we see that $\left|L\left(f^{m}\right)\right|$ is unbounded, a contradiction. Hence $\left|\lambda_{i}\right| \leqq 1$ for all $i$.

LEMMA 6. Given a map $f: T^{n} \rightarrow T^{n}, n \geqq 1$, such that 1 is not a characteristic root of $H_{1}(f): H_{1}\left(T^{n}\right) \rightarrow H_{1}\left(T^{n}\right)$, and $L\left(f^{m}\right), m=1,2, \cdots$, are bounded. Then each nonzero characteristic root of $H_{1}(f)$ is a root of unity.

Proof. Let $\lambda_{1}, \cdots, \lambda_{n}$ be the characteristic roots of $H_{1}(f)$. By Lemma 5 we know that $\left|\lambda_{i}\right| \leqq 1$ for all $i$.

Next we will show that for each $i,\left|\lambda_{i}\right|=0$ or 1 . Suppose not. Let $U=\left\{i \mid \lambda_{i} \neq 0\right\}$, and $q=\# U$. Let

$$
P(\lambda)=\prod_{i=1}^{n}\left(\lambda-\lambda_{i}\right)=\lambda^{n}+a_{1} \lambda^{n-1}+\cdots+a_{n}
$$

be the characteristic polynomial of $H_{1}(f)$. Then $a_{q}=\Pi_{i \in U}\left(-\lambda_{i}\right) \neq 0$. 
Since $\alpha_{q}$ is an integer, we have

$$
1 \leqq\left|a_{q}\right|=\prod_{i \in U}\left|\lambda_{i}\right|<1
$$

because $\left|\lambda_{i}\right| \leqq 1$ for all $i$, and $0<\left|\lambda_{i}\right|<1$ for some $i$. This contradiction shows that for each $i,\left|\lambda_{i}\right|=0$ or 1 .

Note that $a_{m}=0$ for $m>q$, and so

$$
P(\lambda)=\lambda^{n-q}\left(\lambda^{q}+a_{1} \lambda^{q-1}+\cdots+a_{q}\right)=\lambda^{n-q} Q(\lambda) .
$$

All the roots of $Q(\lambda)$ have unit modulus. It is known, [4] page 122, that if all the roots of a monic polynomial with integer coefficients have unit modulus, then they all are roots of unity. This completes the proof.

\section{Geometric lemmas.}

LEMma 7. Suppose the finite group $G$ acts smoothly on a compact manifold $M$, and that $P=\{x \in M \mid g x=x$ for some $g \in G, g \neq 1\}$ is finite. Then there exists a Morse function $\varphi: M \rightarrow \boldsymbol{R}$ such that $\varphi \circ g=\varphi$ for all $g \in G$. Furthermore, each $x \in P$ is a critical point of $\varphi$.

Proof. Following Milnor [5], we will say that a smooth map $f: M \rightarrow \boldsymbol{R}$ is "good" on a set $S \subset M$ if $f$ has no degenerate critical points on $S$.

We begin by obtaining a first approximation, a smooth map $\Psi: M \rightarrow \boldsymbol{R}$, which is invariant (i.e., $\Psi \circ g=\Psi$ for all $g \in G$ ) and is good on a neighborhood $V$ of $P$. Then we perturb $\Psi$ equivariently to the desired Morse function $\varphi$.

It is easy to define a smooth map $h: M \rightarrow R$ such that each $x \in P$ is a nondegenerate critical point of index zero, i.e., in a local coordinate system about $x$ the first partial derivatives vanish and the matrix of second partial derivatives is positive definite at $x$. These same conditions also hold for each $h \circ g, g \in G$, and consequently $\Psi=\sum_{g \in G} h \circ g$ is a first approximation as desired. Clearly, the set $V$ where $\Psi$ is good, is open, contains $P$, and satisfies $g(V)=V$ for all $g \in G$.

Note that $g x \neq x$ for all $x \in M-V$ and $g \in G, g \neq 1$. Now a rather straightforward equivarient version of the argument used in Milnor [5], Theorem 2.7, to prove the existence of Morse functions serves to show that $\Psi$ can be perturbed to an equivarient Morse function $\varphi$. A sketch of this equivarient version follows.

We may find coordinate neighborhoods $U_{1}, \cdots, U_{r}$ such that

$$
M-V \subset \bigcup_{i=1}^{r} U_{i}, \quad P \cap \operatorname{cl}\left(\bigcup_{i=1}^{r} U_{i}\right)=\varnothing, \quad \text { and } \quad U_{i} \cap g\left(U_{i}\right)=\varnothing
$$


for $1 \leqq i \leqq r$ and $g \in G, g \neq 1$. Then $g^{\prime}\left(U_{i}\right) \cap g\left(U_{i}\right)=\varnothing$ for $1 \leqq i \leqq r$ and $g, g^{\prime} \in G, g \neq g^{\prime}$. We can also find compact sets $C_{i} \subset U_{i}$ such that $C_{1}, \cdots, C_{r}$ cover $M-V$. We may alter $\Psi$ in stages so that at the $i$ th stage, the new $\Psi$ is still equivarient and is good on

$$
V \cup\left(\bigcup_{j=1}^{i} \bigcup_{g \in G} g\left(C_{j}\right)\right) \text {. }
$$

At the $i$ th step we simply apply the procedure used in the proof of Theorem 2.7 of [5] to $U_{i}$ and then alter $\Psi$ on $g\left(U_{i}\right)$, for $g \in G, g \neq 1$, so as to preserve the property $\Psi \circ g=g$ for all $g \in G$.

LEMMA 8. Suppose the finite group $G$ acts smoothly on a compact connected m-dimensional manifold $M, m \geqq 2$, and that $P=$ $\{x \in M \mid g x=x$ for some $g \in G, g \neq 1\}$ is finite. Given a finite set $S \subset M-P$ and a point $x_{0} \in M$ such that $g x_{0}=x_{0}$ for all $g \in G$. Then there exists a smooth embedding $\Psi: D^{m} \rightarrow(M-P) \cup\left\{x_{0}\right\}$ such that $\Psi(0)=x_{0}, S \subset \Psi\left(\right.$ int $\left.D^{m}\right)$, and $g\left(\Psi\left(D^{m}\right)\right)=\Psi\left(D^{m}\right)$ for all $g \in G$. Furthermore, for each $g \in G, \Psi^{-1} \circ g \circ \Psi: D^{m} \rightarrow D^{m}$ is the restriction to $D^{m}$ of an orthogonal linear map.

Proof. We will use induction on ${ }^{\#} S$. First suppose $S=\varnothing$. It is an easy matter to embed $M$ into $\boldsymbol{R}^{n}$ for some $n$ such that for each $g \in G$ the map $x \rightarrow g x, x \in M$, is the restriction to $M$ of an orthogonal map $L_{g}: \boldsymbol{R}^{n} \rightarrow \boldsymbol{R}^{n}$. Just start with a smooth embedding $h: M \rightarrow \boldsymbol{R}^{k}$, for some $k$. Set $E_{g}=\boldsymbol{R}^{k}$ for each $g \in G$, and define $e: M \rightarrow \prod_{g \in G} E_{g}=\boldsymbol{R}^{k \|^{\sharp} G}$ by $e(x)=\Pi_{g \in G} h(g x)$, for all $x \in M$. Then the maps $e(x) \rightarrow e(g x), x \in M$ are restrictions of maps $L_{g}: \boldsymbol{R}^{k^{\sharp} G} \rightarrow \boldsymbol{R}^{k^{\sharp} G}$ which simply permute the coordinates of $\boldsymbol{R}^{k^{\sharp \sharp} G}$.

Set $n=k^{\sharp} G$, and identify $M$ with $e(M)$ via $e$. Let $T M_{x}$ be the tangent space of $M$ at $x$, considered as a subspace of $R^{n}$. Let $T=$ $x_{0}+T M_{x_{0}}$ be the geometric tangent space through the point $x_{0}$. Let $N: R^{n} \rightarrow T$ be the orthogonal projection onto $T$. Then $L_{g}(T) \subset T$ for all $g \in G$, and so $L_{g} \circ N=N \circ L_{g}$ for all $g \in G$. The restriction of $N$ to a neighborhood of $x_{0}$ in $M$ is a diffeomorphism onto a neighborhood $W$ of $x_{0}$ in $T$. The desired $\Psi$ is now easily constructed from the restriction of $(N \mid W)^{-1}$ to a ball about $x_{0}$.

Now assume that $\Psi: D^{m} \rightarrow M$ satisfies Lemma 8 as stated. We will show that for any $x \in M-(P \cup S), \Psi$ can be altered so as to satisfy Lemma 8 with $S$ replaced by $S \cup\{x\}$. We will use a connectedness argument. Since $\operatorname{dim} M \geqq 2$ and $P \cup S$ is finite, the space $M-P \cup S$ is connected. Let $V=\{x \in M-P \cup S \mid$ Lemma 8 holds with $S$ replaced by $S \cup\{x\}$.

The set $V$ is clearly open in $M-(P \cup S)$. We will show that $(M-(P \cup S))-V$ is also open. Let $x \in(M-(P \cup S))-V$. If $g x \in S$ 
for some $g \in G$, then $\Psi$ already satisfies $S \cup\{x\} \subset \Psi\left(\right.$ int $\left.D^{m}\right)$. Hence $g x \notin S$ for all $g \in G$. Since $g x \neq x$ for all $g \in G, g \neq 1$, we may find a coordinate neighborhood $U$ of $x$ which is diffeomorphic to an open $m$-ball and such that $g(U) \subset M-(P \cup S)$, and $g(U) \cap U=\varnothing$ for all $g \in G, g \neq 1$. Then $g(U) \cap h(U)=\varnothing$ for $g, h \in G, g \neq h$. We claim that $U \subset(M-(P \cup S))-V$. Suppose not. Then we can find a $y \in U \cap V$. Let $k: M \rightarrow M$ be a diffeomorphism which is fixed outside of $U$ and $k(y)=x$. Define $\bar{k}: M \rightarrow M$ by

$$
\bar{k}(p)=\left\{\begin{array}{cl}
p & \text { if } p \notin \bigcup_{g \in G} g(U) \\
g k g^{-1}(p) & \text { if } p \in g(U) .
\end{array}\right.
$$

Then $\bar{k}$ is a well defined diffeomorphism, and Lemma 8 is satisfied with $\Psi$ replaced by $\bar{k} \circ \Psi$ and $S$ replaced by $S \cup\{x\}$. In fact, for each $g \in G, \bar{k} \circ g=g \circ \bar{k}$ and so $(\bar{k} \circ \Psi)^{-\imath} \circ g \circ(\bar{k} \circ \Psi)=\Psi^{-1} \circ g \circ \Psi$. This proves the claim, and hence $(M-(P \cup S))-V$ is open.

Since $V \neq \varnothing$, we have $V=M-(P \cup S)$, and the induction step is complete.

\section{Proofs of the theorems.}

Proof of Theorem 4. First recall that a flow on $M$ is a smooth map $F: M \times R \rightarrow M$ such that with the notation $F_{t}(x)=F(x, t)$ we have $F_{s} \circ F_{t}=F_{s+t}$ for all $s, t \in R$, and $F_{0}=1_{M}$. An orbit of $F$ is a function of the form $F^{x}: \boldsymbol{R} \rightarrow M$ where $x \in M$ and $F^{x}(t)=F(x, t)$ for all $t \in \boldsymbol{R}$. An orbit $F^{x}$ is periodic if $F^{x}(s)=F^{x}(0)$ for some $s \neq 0$. Thus the constant orbits are considered to be periodic.

The idea of the proof is to obtain a flow $H_{t}: M \rightarrow M$ such that the orbits $H^{x}$ for $x \in P$ are constants and these are the only periodic orbits, and $H_{t} \circ f=f \circ H_{t}$. Then $g=H_{1} \circ f$ will satisfy the conclusions of the theorem. The desired flow $H_{t}$ is obtained in several steps.

Since $f^{N}=1$, we have a smooth action of $\boldsymbol{Z} / n \boldsymbol{Z}$ on $M$. Let $\varphi: M \rightarrow \boldsymbol{R}$ be the Morse function given by Lemma 7 . It is easy to obtain an equivarient Riemannian metric on $M$. Just average over $\boldsymbol{Z} / n \boldsymbol{Z}$ any Riemannian metric. Then the gradient of $\phi$ with respect to this equivarient Riemannian metric is an equivarient vector field $v$. The vector field $v$ determines a flow $F$ on $M$ which satisfies $F_{t}(f(x))=f\left(F_{t}(x)\right)$ for all $(x, t) \in M \times R$. The flow also satisfies $\varphi\left(F_{t}(x)\right)>\varphi(x)$ whenever $x$ is not a critical point of $\varphi$ and $t>0$. Hence the only periodic orbits of $F$ are the constant orbits at critical points of $\varphi$.

Let $S=\{x \in M \mid x$ is a critical point of $\varphi\}-P$. Let $\Psi: D^{m} \rightarrow M$ be given by Lemma 8. Pick $r \in(0,1)$ such that $S \subset \Psi\left(D_{r}^{m}\right)$, where $D_{r}^{m}=\left\{x \in \boldsymbol{R}^{m} \mid\|x\| \leqq r\right\}$. Let $b: \boldsymbol{R} \rightarrow \boldsymbol{R}$ be a smooth map satisfying 
$b(t)=0$ for $t \leqq r, b(t)>0$ for $t>r$, and $b(t)=1$ for $t \geqq(1+r) / 2$. Define $\bar{b}: M \rightarrow \boldsymbol{R}$ by

$$
\bar{b}(x)=\left\{\begin{array}{cl}
1 & \text { if } x \notin \Psi\left(D_{(r+1) / 2}^{m}\right) \\
b\left(\left\|\Psi^{-1}(x)\right\|\right) & \text { if } x \in \Psi\left(D^{m}\right)
\end{array}\right.
$$

It is clear that $\bar{b}$ is well defined and smooth. Define a new vector field $w$ by $w(x)=\bar{b}(x) v(x)$. Then $w$ is equivarient under $f$ and determines an equivarient flow $G_{t}$. The orbits of $G$, which are just the integral curves of $w$, are reparameterizations of portions of orbits of $F$. The orbits $G^{x}$ for $x \in \Psi\left(D_{r}^{m}\right) \cup P$ are constant. All the other orbits are reparameterizations of portions of nonperiodic orbits of $F$ by reparameterization functions which are strictly monotone increasing functions. Hence the orbits $G^{x}$ for $x \in \Psi\left(D_{r}^{m}\right) \cup P$ are the only periodic orbits. Let $\theta: \boldsymbol{R} \rightarrow \boldsymbol{R}$ be a smooth map satisfying $\theta(t)=r+t$ for $t \leqq 1 / 3(1-r), \theta^{\prime}(t)>0$ for all $t$, and $\theta(t)=t$ for $t \geqq r+2 / 3(1-r)$. We will use later the obvious fact that $\theta^{-1}: \boldsymbol{R} \rightarrow \boldsymbol{R}$ exists and is smooth. Define $h: M-\left\{x_{0}\right\} \rightarrow M-\Psi\left(D_{r}^{m}\right)$ by

$$
h(x)=\left\{\begin{array}{cl}
x & \text { if } x \notin \Psi\left(D^{m}\right) \\
\frac{\theta(\|x\|) x}{\|x\|} & \text { if } x \in \Psi\left(D^{m}\right),
\end{array}\right.
$$

where we have identified $\Psi\left(D^{m}\right)$ with $D^{m}$ via $\Psi$. Define $H_{t}(x)$ by

$$
H_{t}(x)=\left\{\begin{array}{cc}
h^{-1}\left(G_{t}(h(x))\right) & \text { for } x \neq x_{0} \\
x_{0} & \text { for } x=x_{0}
\end{array} .\right.
$$

We wish to show that $H_{t}(x)$ is a smooth flow by showing that $H_{t}(x)$ is determined by a smooth vector field. Since $H_{s} \circ H_{t}=H_{s+t}$, and $H_{0}=1_{M}$, it is sufficient to show that $\eta(x)=d / d t H_{t}(x)$ at $t=0$ is a smooth vector field. It is clear that $\eta(x)$ is well defined for all $x \in M$ and $\bar{\eta}=\eta \mid M-\left\{x_{0}\right\}$ is smooth. Since $\eta\left(x_{0}\right)=0$, it is sufficient to show that $\eta(x)$ and all its derivatives approach 0 as $x \rightarrow x_{0}$. We calculate for $x \neq x_{0}$

$$
\begin{aligned}
\eta(x) & =\left.\frac{d}{d t}\left(H_{t}(x)\right)\right|_{t=0}=\left.\left.d h^{-1}\right|_{\sigma_{0}(h(x))} \frac{d}{d t}\left(G_{t}(h(x))\right)\right|_{t=0} \\
& =\left.d h^{-1}\right|_{h(x)} w(h(x)) .
\end{aligned}
$$

Since $w$ and all its derivatives vanish on $D_{r}^{m}$, Taylor expansions show that for each derivative $a(x)$ of a component of $w(x)$ and each $n \geqq 1$ there is a constant $c$ such that

$$
|a(x)| \leqq c|\|x\|-r|^{n} \quad \text { for } x \in \Psi\left(D^{m}\right) .
$$


The form of $h(x)$ for $x \in \Psi\left(D^{m}\right)-\left\{x_{0}\right\}$ and $\|x\| \leqq(1-r) / 3$, is

$$
h(x)=\frac{(r+\|x\|) x}{\|x\|}
$$

and hence if $u(x)$ is a derivative of a component of $h$ of order $n$, then there is a constant $e$ such that

$$
|u(x)| \leqq e\|x\|^{-n-1} \quad \text { for } 0<\|x\| \leqq \frac{1-r}{3} .
$$

The map $h^{-1} \mid\left(\Psi\left(D^{m}\right)-\Psi\left(D_{r}^{m}\right)\right)$ has a smooth extention $\bar{h}^{-1}: \varphi\left(D^{m}\right)-$ $\left\{x_{0}\right\} \rightarrow \varphi\left(D^{m}\right)$ given by $\bar{h}^{-1}(y)=\theta^{-1}(\|y\|) y /\|y\|$. Consequently $\left.d h^{-1}\right|_{y}$ and all its derivatives are bounded. Using this and (5) and (6) we see that $\eta(x)=\left.d h^{-1}\right|_{h(x)} w(h(x))$ and all its derivatives approach 0 as $x \rightarrow x_{0}$. Hence $\eta(x)$ is smooth and therefore so is $H_{t}(x)$.

It is clear from the definition of $H_{t}(x)$ and the properties of $G_{t}(x)$ that the only periodic orbits of $H$ are the constant orbits $H^{x}$ for $x \in P$. It is also clear from the fact that $x \rightarrow \Psi^{-1}(f(\Psi(x)))$ is the restriction of a orthogonal linear map to $D^{m}$, that $f \circ h=h \circ f$ and hence $f \circ H_{t}=H_{t} \circ f$ for all $t$. The map $(x, t) \rightarrow H_{t}(f(x))$ is a homotopy from $H_{0} \circ f=f$ to the smooth map $g=H_{1} \circ f$. It is easy to see that the set of periodic points of $H_{1} \circ f$ is $P$ and that $H_{1} \circ f|P=f| P$. This completes the proof.

Lemma 9. Suppose there is given a map $g: T^{k} \rightarrow T^{k}, k \geqq 2$, which is covered by a linear map $A: \boldsymbol{R}^{k} \rightarrow \boldsymbol{R}^{k}$, and an integer $N \geqq 2$, such that $g^{N}=1_{T^{k}}$, and $\lambda^{m} \neq 1$ for $\lambda$ a characteristic root of $A$ and $1 \leqq$ $m<N$. Then there exists a smooth map $\bar{g}$ homotopic to $g$ such that $P=\left\{x \in T^{k} \mid g^{m}(x)=x\right.$ for some $\left.m, 1 \leqq m<N\right\}=$ the set of all periodic points of $\bar{g}, \bar{g}|P=g| P$, and for $m \geqq 1 \#\left\{x \in T^{k} \mid \bar{g}^{m}(x)=x\right\} \leqq a_{m}(g)$.

Proof. We wish to apply Theorem 4. If follows from Lemma 2 that $P=\left\{x \in T^{k} \mid g^{r}(x)=x\right.$ for some $\left.r, 1 \leqq r<N\right\}$ is finite. Since $N \geqq 2, \lambda_{i}^{1} \neq 1$ for all characteristic roots $\lambda_{i}$ and hence

$$
L(g)=\prod_{i=1}^{n}\left(1-\lambda_{i}\right) \neq 0
$$

by formula (*). Hence, we can find an $x_{0} \in T^{k}$ such that $g\left(x_{0}\right)=x_{0}$. Therefore Theorem 4 gives a smooth map $\bar{g}$ homotopic to $g$ such that $P=$ the set of periodic points of $\bar{g}$, and $\bar{g}|P=g| P$. It follows from Lemma 2 applied to $g$, and formula $(*)$, that for $1 \leqq m<N$,

$$
\#\left\{x \in T^{k} \mid \bar{g}^{m}(x)=x\right\}=\#\left\{x \in T^{k} \mid g^{m}(x)=x\right\}=\left|L\left(g^{m}\right)\right|=a_{m}(g) .
$$

Then 


$$
\begin{aligned}
\#\{x & \left.\in T^{k} \mid \bar{g}^{N}(x)=x\right\} \\
& =\sum_{m<N} \#\left\{x \in T^{k} \mid x \text { is a periodic point of } \bar{g} \text { of period } m\right\} \\
& \leqq \sum_{m \backslash N}\left|L\left(g^{m}\right)\right| \\
& =\sum_{m \mid N}\left|\prod_{i=1}^{k}\left(1-\lambda_{i}^{m}\right)\right| \\
& \leqq \prod_{i=1}^{k} \sum_{m \mid N}\left|1-\lambda_{i}^{m}\right| \\
& \leqq \prod_{i=1}^{k} a_{i N} \\
& =a_{N}(g) .
\end{aligned}
$$

To complete the proof it is sufficient to show that if $m \geqq 1, q \geqq 1$, and $m \equiv q \bmod N$, then

$$
\left\{x \in T^{k} \mid \bar{g}^{m}(x)=x\right\}=\left\{x \in T^{k} \mid \bar{g}^{q}(x)=x\right\} .
$$

We may assume $m<q$ and so $q=m+p N$ for some $p \geqq 1$. Assume $\bar{g}^{m}(x)=x$. Then $x \in P$ and so $\bar{g}^{N}(x)=g^{N}(x)=x$. Consequently $\bar{g}^{q}(x)=\bar{g}^{m+p N}(x)=\bar{g}^{m}(x)=x$. The reverse implication, " $\bar{g}^{q}(x)=x$ implies $\bar{g}^{m}(x)=x$ " follows similarly. This completes the proof.

REMARK. In our application of Lemma 9 in the proof of Theorem 3, Lemma 9 needs to be augmented by the following observation. Lemma 9 also holds when $k=1$ and $N \geqq 1$. We verify this as follows. It is easy to deduce that $A=1_{R^{1}}$ or $A=-1_{R^{1}}$. In the first case, $A=$ $1_{R^{1}}$, we can homotopy $g=1_{T^{1}}$ to a rotation $\bar{g}$ of the circle $S^{1}=T^{1}$ by an angle which has an irrational ratio to $2 \pi$. Such a $\bar{g}$ has no periodic points and Lemma 9 is verified in this case.

In the second case, $A=-1_{R^{1}}, g$ is a reflection and there are exactly two fixed points $x_{0}$ and $x_{1}$. It is easy to homotopy $g$ to a map $\bar{g}$ which leaves $x_{0}$ and $x_{1}$ fixed, and moves all other points away from $x_{0}$ and closer to $x_{1}$. Then $x_{0}$ and $x_{1}$ will be the only periodic points of $\bar{g}$. It is easy to calculate that $a_{m}(g)=2$ for all $m \geqq 1$, and so Lemma 9 holds in this case also.

Proof of Theorem 3. We prove Theorem 3 by induction on $n$. By convention $\boldsymbol{R}^{0}$ and $T^{0}$ are singletons. Hence the case $n=0$ holds trivially. Now assume that $n>0$ and the theorem holds for all $m<n$. Let $f: T^{n} \rightarrow T^{n}$ be a map.

By Lemma 1 we may assume that $f$ is covered by a linear map $F: \boldsymbol{R}^{n} \rightarrow \boldsymbol{R}^{n}$. By Lemma 5 we have 


$$
L\left(f^{m}\right)=\prod_{j=1}^{n}\left(1-\lambda_{i}^{m}\right)
$$

where $\lambda_{1}, \cdots, \lambda_{n}$ are the characteristic roots of $F$.

First consider the case where $\lambda_{i}^{m} \neq 1$ for all $m \geqq 1$ and $1 \leqq i \leqq n$. Then the theorem follows from Lemmas 2 and 5 .

Consider now the remaining case where $\lambda_{i}^{m}=1$ for some $m \geqq 1$ and $1 \leqq i \leqq n$. Let $N$ be the smallest such $m$. Let $\bar{F}^{t}$ denote the transpose of $\bar{F}$. Since $\left(\bar{F}^{t}\right)^{N}$ has integer entries, we may find a $w \in \boldsymbol{Z}^{n}$ such that $w \neq 0$ and $\left(\bar{F}^{t}\right)^{N} w=w$. Set

$$
W=\operatorname{sp}_{\boldsymbol{R}^{n}}\left\{\left(\bar{F}^{t}\right)^{m} w \mid 0 \leqq m<N\right\} .
$$

Then $\operatorname{dim} W \geqq 1, \bar{F}^{t} u \in W$ for all $u \in W$, and $\left(\bar{F}^{t}\right)^{N} x=x$ for all $x \in W$. Set $k=\operatorname{dim} W$. By Lemma 4 , we can find a basis $w^{1}, w^{2}, \cdots, w^{n}$ for $\boldsymbol{Z}^{n}$ such that $w^{1}, w^{2}, \cdots, w^{k}$ form a basis for $W$. Let $K: \boldsymbol{R}^{n} \rightarrow \boldsymbol{R}^{n}$ be the linear transformation whose matrix satisfies $\bar{K}^{t} e^{i}=w^{i}$. Then, both $\bar{K}$ and $\bar{K}^{-1}$ have integer entries. Thus, both $K$ and $K^{-1}$ induce maps $K^{\prime}: T^{n} \rightarrow T^{n}$, and $K^{-1 \prime}=K^{\prime-1}: T^{n} \rightarrow T^{n}$. We "change coordinates" by noting that it is sufficient to prove the theorem for

$$
g=K^{\prime} \circ f \circ K^{\prime-1}
$$

in place of $f$. The map $g$ is covered by

$$
M=K \circ F \circ K^{-1}: \boldsymbol{R}^{n} \longrightarrow \boldsymbol{R}^{n} .
$$

From $\bar{K}^{t} e^{i}=w^{i}$ and $\bar{F}^{t} u \in W$ for $u \in W$, it follows that the matrix $\bar{M}$ of $M$ has the form

$$
\bar{M}=\left(\begin{array}{c|c}
\bar{A} & 0 \\
\hline \bar{C} & \bar{B}
\end{array}\right)
$$

where $\bar{A}, \bar{B}, \bar{C}$, and 0 are $k \times k,(n-k) \times(n-k),(n-k) \times k$ and $n \times(n-k)$ matrices, and all entries of 0 are zero. It follows from $\bar{F}^{t^{N}} x=x$ for all $x \in W$, that $\bar{A}^{N}=1$. Since $\bar{M}=\bar{K} \cdot \bar{F} \cdot \bar{K}^{-1}$ is similar to $\bar{F}, \lambda_{1}, \cdots, \lambda_{n}$ are the characteristic roots of $\bar{M}$. Hence, we may renumber the $\lambda_{i}$ 's so that $\lambda_{1}, \cdots, \lambda_{k}$ and $\lambda_{k+1}, \cdots, \lambda_{n}$ are the characteristic roots of $\bar{A}$ and $\bar{B}$ respectively. Let

$$
B: \boldsymbol{R}^{n-k} \longrightarrow \boldsymbol{R}^{n-k}
$$

be the linear map whose matrix is $\bar{B}$. Then $B$ induces a map $b: T^{n-k} \rightarrow T^{n-k}$. Since $k=\operatorname{dim} W \geqq 1$, we may apply our induction hypothesis to $b$ and find a smooth map $\bar{b}$ homotopic to $b$ such that

$$
\#\left\{x \in T^{n-k} \mid \bar{b}^{m}(x)=x\right\} \leqq a_{m}(b)=\prod_{i=k+1}^{n} a_{i m} .
$$


Let $A: \boldsymbol{R}^{k} \rightarrow \boldsymbol{R}^{k}$ be the linear map whose matrix is $\bar{A}$. Let $a: T^{k} \rightarrow T^{k}$ be the map induced by $A$. Since $\bar{A}^{N}=1$, we have $a^{N}=1_{T^{k}}$. Because we choose $N$ so that $\lambda_{i}^{m} \neq 1$ for $1 \leqq m<N$ and all $i$, and $N=1$ implies $k=1$, we see that Lemma 9 or the remark which follows it applies. Hence we can find a smooth map $\bar{a}$ homotopic to $a$ such that $P=\left\{x \in T^{k} \mid a^{m}(x)=x\right.$ for some $\left.m, 1 \leqq m<N\right\}=$ the set of all periodic points of $\bar{a}, \bar{a}|P=a| P$, and for $m \geqq 1$,

$$
\#\left\{x \in T^{k} \mid \bar{a}^{m}(x)=x\right\} \leqq a_{m}(a)=\prod_{i=1}^{k} a_{i m} .
$$

If we write $\boldsymbol{R}^{n}=\boldsymbol{R}^{k} \times \boldsymbol{R}^{n-k}$, then $M$ has the form

$$
M(x, y)=(\bar{A} x, \bar{C} x+\bar{B} y) .
$$

Consequently, if we write $T^{n}=T^{k} \times T^{n-k}$, then $g(u, v)=(a(u), r(u, v))$ where $r: T^{k} \times T^{n-k} \rightarrow T^{k}$ is the map induced by the map $R: \boldsymbol{R}^{k} \times \boldsymbol{R}^{n-k} \rightarrow$ $\boldsymbol{R}^{k}$ which is given by $R(x, y)=\bar{C} x+\bar{B} y$. The homotopy from $a$ to $\bar{a}$ gives rise to a homotopy from $g$ to $\bar{g}$ where

$$
\bar{g}(u, v)=(\bar{a}(u), r(u, v)) .
$$

The periodic points of $\bar{g}$ must have the form

$$
(u, v) \in T^{k} \times T^{n-k} \quad \text { where } u \in P .
$$

Partition $P$ into orbits under $\bar{a}$. Let

$$
X=\left\{u_{i}=\bar{a}^{i}\left(u_{0}\right) \mid i=0,1, \cdots, m-1\right\}
$$

be one such orbit consising of $m$ distinct point, where $1 \leqq m<N$ and $\bar{a}^{m}\left(u_{0}\right)=u_{0}$. Consider the maps

$$
g_{i}=\bar{g} \mid u_{i} \times T^{n-k}: u_{i} \times T^{n-k} \longrightarrow u_{i+1} \times T^{n-k},
$$

which are covered by the maps

$$
M_{i}=M \mid x_{\imath} \times \boldsymbol{R}^{n-k}: x_{i} \times \boldsymbol{R}^{n-k} \longrightarrow x_{i+1} \times \boldsymbol{R}^{n-k}
$$

where $u_{m}=\bar{a}^{m}\left(u_{0}\right)=u_{0}$ and $x_{0}$ is chosen so that $\pi\left(x_{0}\right)=u_{0}$, and $x_{i}=$ $\bar{A}^{i} x_{0}$ for $i \geqq 1$, (recall that $\bar{a}|P=a| P$ ). Making the obvious identifications of $u_{i} \times T^{n-k}$ with $T^{n-k}$, and $x_{i} \times \boldsymbol{R}^{n-k}$ with $\boldsymbol{R}^{n-k}$ we see that

$$
M_{i}(y)=\bar{C} x_{i}+\bar{B} y
$$

for all $y \in \boldsymbol{R}^{n-k}$. Define

$$
M_{i t}(y)=t \bar{C} x_{\imath}+\bar{B} y .
$$

Then $M_{i 1}=M_{i}$ and $M_{i 0}$ has $\bar{B}$ as its matrix. Because $\bar{B}$ has integer entries and $t \bar{C} x_{i}$ does not depend on $y$, the homotopy $M_{i t}$ induces a 
homotopy $g_{i t}$ from $g_{i 1}=g_{i}$ to the map induced by $M_{i 0}$, which is $b$. Since $b$ is homotopic to $\bar{b}$, each $g_{i}$ is homotopic to $\bar{b}$. Since both $g_{i}$ and $\bar{b}$ are smooth, we may find a smooth homotopy $h_{i}: T^{n-k} \times I \rightarrow$ $T^{n-k}$ such that for some $\varepsilon>0, h_{i}(v, t)=\bar{b}(v)$ for all $t<\varepsilon$, and $h_{i}(v, t)=$ $g_{i}(v)=r\left(u_{i}, v\right)$ for all $t>1-\varepsilon$.

Pick coordinate charts $\left(U_{i}, \varphi_{i}\right)$ about the points $u_{i}$ such that $\left\{u_{i}\right\}=$ $U_{i} \cap P, \varphi_{i}\left(U_{i}\right)=B_{1}(0) \subset \boldsymbol{R}^{k}$, and $\varphi_{i}\left(u_{i}\right)=0$. Using the natural group structure on $T^{n-k}$ we define

$$
r_{t}: T^{k} \times T^{n-k} \longrightarrow T^{n-k} \quad \text { for } t \in[0,1]
$$

by

$$
r_{t}(u, v)=\left\{\begin{array}{cl}
r(u, v)+h_{i}\left(v, t\left\|\varphi_{i}(u)\right\|+1-t\right)-r\left(u_{i}, v\right) & \text { if } u \in U_{i} \\
r(u, v) & \text { if } u \notin \bigcup_{i=1}^{m} U_{i} .
\end{array}\right.
$$

Using $r_{t}$ we obtain a homotopy

$$
\bar{g}_{t}(u, v)=\left(\bar{a}(u), r_{t}(u, v)\right)
$$

from $\bar{g}_{0}=\bar{g}$ to $\bar{g}_{1}$, where $\bar{g}_{1}(u, v)=\left(\bar{a}(u), r_{1}(u, v)\right)$. Note that $r_{1}\left(u_{i}, v\right)=$ $\bar{b}(v)$ for each $i=0,1, \cdots, m-1$. Proceed similarly with the other orbits in $P$ and call the final map $\widetilde{g}$.

The map $\widetilde{g}$ will be smooth and homotopic $\bar{g}$ and hence homotopic to $g$. For all $(u, v) \in T^{k} \times T^{n-k}, \widetilde{g}(u, v) \in(\bar{a}(u), \widetilde{r}(u, v))$ for some map $\widetilde{r}: T^{k} \times T^{n-k} \rightarrow T^{n-k}$ which satisfies $\tilde{r}(u, v)=\bar{b}(v)$ for all $u \in P$.

Now suppose $\widetilde{g}^{m}(u, v)=(u, v)$. Then $\bar{a}^{m}(u)=u$ and so $u \in P$. Hence $\bar{a}^{i}(u) \in P$ for all $i$ and so by an easy induction $\widetilde{g}^{i}(u, v)=$ $\left(\bar{a}^{i}(u), \bar{b}^{i}(v)\right)$. Applying this with $i=m$ we see that $\bar{b}^{m}(v)=v$. Hence

$$
\begin{aligned}
& \#\left\{(u, v) \in T^{k} \times T^{n-k} \mid \widetilde{g}^{m}(u, v)=(u, v)\right\} \\
& \quad \leqq \#\left\{u \in T^{k} \mid \bar{a}^{m}(u)=u\right\} \times \#\left\{v \in T^{n-k} \mid b^{m}(v)=v\right\} \\
& \quad \leqq \prod_{i=1}^{k} a_{i m} \cdot \prod_{i=k+1}^{n} a_{i m}=a_{m} .
\end{aligned}
$$

This completes the proof.

Proof of Theorem 1. The "if" direction follows from the Nielsen fixed point theorem and Theorem 2.

Next we prove the converse direction. Assume that $L\left(f^{m}\right), m=$ $1,2, \cdots$, are bounded. We may assume $n \geqq 1$. Let $g$ be the map given by Theorem 3 . If 1 is a characteristic root of $H_{1}(f): H_{1}\left(T^{n}\right) \rightarrow$ $H_{1}\left(T^{n}\right)$, then $g$ has no periodic points because $a_{m}(f)=0$ for all $m \geqq 1$. So assume that 1 is not a characteristic root of $H_{1}(f)$. Now from Lemma 6 we have $\left|\lambda_{i}\right| \leqq 1$ for all $i$, where $\lambda_{1}, \cdots, \lambda_{n}$ are the characteristic roots of $H_{1}(f)$. Consequently, there exists a $B$ such that 
$a_{i m} \leqq B$ for all $i=1, \cdots, n$, and $m \geqq 1$. Thus $a_{m}(f) \leqq B^{n}$ for all $m \geqq 1$.

We will show that the number of periodic points of $g$ is bounded by $B^{n}$. Suppose on the contrary that $S=\left\{x_{i} \mid 1 \leqq i \leqq B^{n}+1\right\}$ is a set of $B^{n}+1$ distinct periodic points such that $x_{\imath}$ has period $m_{i}$. Set

$$
m=\prod_{i=1}^{B n+1} m_{i} .
$$

Then $S \subset\left\{x \in T^{n} \mid g^{m}(x)=x\right\}$. But, by Theorem 3,

$$
\#\left\{x \in T^{n} \mid g^{m}(x)=x\right\} \leqq a_{m}(f) \leqq B^{n},
$$

a contradiction. This completes the proof.

\section{REFERENCES}

1. R. Brooks, R. Brown, J. Pak, and D. Taylor, Nielsen numbers of maps of tori, Proc. Amer. Math. Soc., 52 (1975), 398-400.

2. R. Brown, The Lefschetz Fixed Point Theorem, Scott, Foresman and Company, 1971.

3. M. Hirsch, Differential Topology, Springer-Verlag, New York, 1976.

4. Hecke E. Leipzig, Theorie der Algebraischen Zahlen, Akademische Verlagsgesellschaft, 1923.

5. J. Milnor, Lectures on the h-cobordism Theorem, Princeton Math. Notes, Princeton, New Jersey, 1965.

Received February 15, 1978 and in revised form December 4, 1978.

INDIANA UNIVERSITY

BLOomington, IN 47401 



\section{PACIFIC JOURNAL OF MATHEMATICS}

EDITORS

DONALD BABBITT (Managing Editor)

University of California

Los Angeles, California 90024

Hugo RossI

University of Utah

Salt Lake City, UT 84112

C. C. MOORE and ANDREW OGG

University of California

Berkeley, CA 94720

\section{J. DUGUNDJI}

Department of Mathematics University of Southern California Los Angeles, California 90007

R. Finn and J. Milgram Stanford University

Stanford, California 94305

\section{ASSOCIATE EDITORS}
E. F. BECKENBACH
B. H. Neumann
F. WOLF
K. YoSHIDA

\section{SUPPORTING INSTITUTIONS}

UNIVERSITY OF BRITISH COLUMBIA

CALIFORNIA INSTITUTE OF TECHNOLOGY

UNIVERSITY OF CALIFORNIA

MONTANA STATE UNIVERSITY

UNIVERSITY OF NEVADA, RENO

NEW MEXICO STATE UNIVERSITY

OREGON STATE UNIVERSITY

UNIVERSITY OF OREGON
UNIVERSITY OF SOUTHERN CALIFORNIA

STANFORD UNIVERSITY

UNIVERSITY OF HAWAII

UNIVERSITY OF TOKYO

UNIVERSITY OF UTAH

WASHINGTON STATE UNIVERSITY

UNIVERSITY OF WASHINGTON 


\section{Pacific Journal of Mathematics}

\section{Vol. 83, No. 1 \\ March, 1979}

Richard Neal Ball, Topological lattice-ordered groups ............... 1

Stephen Berman, On the low-dimensional cohomology of some

infinite-dimensional simple Lie algebras .................. 27

R. P. Boas and Gerald Thomas Cargo, Level sets of derivatives ......... 37

James K. Deveney and John Nelson Mordeson, Splitting and modularly

perfect fields......................................

Robert Hugh Gilman and Ronald Mark Solomon, Finite groups with small

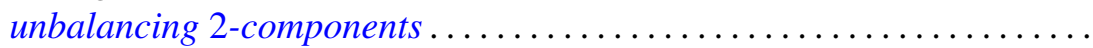

George Grätzer, Andras Hajnal and David C. Kelly, Chain conditions in free products of lattices with infinitary operations..................

Benjamin Rigler Halpern, Periodic points on tori ..................

Dean G. Hoffman and David Anthony Klarner, Sets of integers closed under

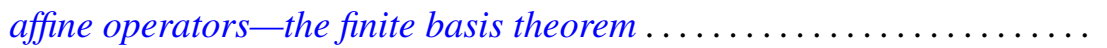

Rudolf-Eberhard Hoffmann, On the sobrification remainder ${ }^{s} X-X \ldots \ldots$

Gerald William Johnson and David Lee Skoug, Scale-invariant

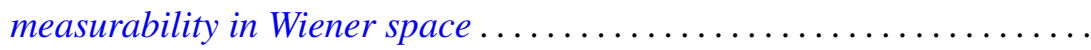

Michael Keisler, Integral representation for elements of the dual of

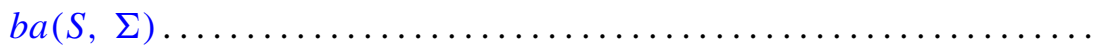

Wayne C. Bell and Michael Keisler, A characterization of the representable Lebesgue decomposition projections ................

Wadi Mahfoud, Comparison theorems for delay differential equations ...

R. Daniel Mauldin, The set of continuous nowhere differentiable functions .

Robert Wilmer Miller and Mark Lawrence Teply, The descending chain condition relative to a torsion theory...

Yoshiomi Nakagami and Colin Eric Sutherland, Takesaki's duality for regular extensions of von Neumann algebras ........ .

William Otis Nowell, Tubular neighborhoods of Hilbert cube manifolds ...

Mohan S. Putcha, Generalization of Lentin's theory of principal solutions of word equations in free semigroups to free product of copies of positive reals under addition

Amitai Regev, A primeness property for central polynomials . ...

Saburou Saitoh, The Rudin kernels on an arbitrary domain. . .

Heinrich Steinlein, Some abstract generalizations of the

Ljusternik-Schnirelmann-Borsuk covering theorem . . . 